\title{
Optimal Timing of Sequencing From Platinum- based Chemotherapy to Pembrolizumab for Advanced Urothelial Carcinoma Based on Real- World Data: A Multi-Institutional Retrospective Study
}

Nobuki Furubayashi ( $\nabla$ furubayashi-jua@umin.ac.jp )

Kyushu Cancer Center https://orcid.org/0000-0001-9159-493X

Futoshi Morokuma

Saga-ken Medical Centre Koseikan

Toshihisa Tomoda

Oita Prefectural Hospital: Oita Kenritsu Byoin

Yoshifumi Hori

Miyazaki Prefectural Miyazaki Hospital: Miyazaki Kenritsu Miyazaki Byoin

Takahito Negishi

Kyushu Cancer Center

Akihiro Miura

Kyushu Cancer Center

Nobutaka Nakamura

Kyushu Cancer Center

Yoshiaki Sato

Kyushu Cancer Center

Noriaki Tokuda

Saga-ken Medical Centre Koseikan

Kentaro Kuroiwa

Miyazaki Prefectural Miyazaki Hospital: Miyazaki Kenritsu Miyazaki Byoin

Motonobu Nakamura

Kyushu Cancer Center

Research article

Keywords: advanced urothelial carcinoma, platinum-based chemotherapy, pembrolizumab, real-world clinical practice 
Posted Date: December 3rd, 2020

DOI: https://doi.org/10.21203/rs.3.rs-116673/v1

License: (c) (i) This work is licensed under a Creative Commons Attribution 4.0 International License. Read Full License 


\section{Abstract}

\section{Background}

To evaluate the optimal timing of sequencing from platinum-based chemotherapy to pembrolizumab with advanced urothelial carcinoma (UC) in real-world clinical practice.

\section{Methods}

A total of 34 patients who received pembrolizumab as second-line treatment after first-line platinumbased chemotherapy for advanced UC at 4 institutions between January 2018 and August 2020 were retrospectively evaluated.

Results

The gemcitabine and cisplatin regimen was administered to 27 patients $(79.4 \%)$, and the gemcitabine and carboplatin regimen was administered to 6 patients (20.6\%). Regarding the number of chemotherapy cycles administered before pembrolizumab, $\leq 4$ cycles were administered to 22 patients $(64.7 \%), 5-6$ cycles to 4 patients $(11.8 \%)$ and $\geq 7$ cycles to 8 patients $(23.5 \%)$. When the number of cycles was divided into 4 courses, the median overall survival (OS) for $\leq 4$ and $>4$ prior chemotherapy cycles was 7.0 months and 25.5 months, respectively $(P=0.034)$. When the number of cycles was divided into 6 courses, the median OS for $\leq 6$ and $>6$ prior chemotherapy cycles was 11.3 months and 6.6 months, respectively $(P=0.658)$. Multivariate analyses for the OS of pembrolizumab revealed that the Eastern Cooperative Oncology Group Performance Status ( $\geq 2$ : hazard ratio $[\mathrm{HR}]$ 9.994, 95\% $\mathrm{Cl}=1.854-53.879, \mathrm{P}=0.007$ ), liver metastases (positive: $\mathrm{HR} 3.751,95 \% \mathrm{Cl}=0.958-13.603, \mathrm{P}=0.047$ ) and time from previous chemotherapy ( $\geq 3$ months: HR 0.094, 95\% $\mathrm{Cl}=0.023-0.357, \mathrm{P}<0.001$ ) were independent prognostic factors, but the number of chemotherapy cycles before pembrolizumab was not an independent prognostic factor. When the number of cycles was divided into 4 courses, the median OS from the first-line treatment with $\leq 4$ and $>4$ prior chemotherapy cycles was 17.3 months and 37.1 months, respectively $(P<0.001)$. When the number of cycles was divided into 6 courses, the median OS from the first-line treatment for $\leq 6$ and $>6$ prior chemotherapy cycles was 18.6 months and 27.3 months, respectively $(P=0.276)$.

Conclusions

These real-world findings suggest that the optimal timing of sequencing from platinum-base chemotherapy to pembrolizumab in advanced UC is around six cycles, even if no exacerbations are observed with first-line chemotherapy.

\section{Background}

Despite recent improvements in the field of oncology, the prognosis for patients with advanced or metastatic urothelial carcinoma (UC) remains dismal. The standard treatment for metastatic UC has long been systemic chemotherapy, predominantly platinum-based regimens of gemcitabine plus cisplatin (GC) 
or cisplatin plus methotrexate, doxorubicin and vinblastine (MVAC) [1-3]. The GC and MVAC regimens showed similar outcomes in terms of the overall survival (OS) in a large, randomized phase 3 study. In addition, the time to treatment failure and overall response rate were not significantly different. However, GC is frequently preferred due to its lower hematological and mucosal toxicity [4].

The renal toxicity of platinum-based chemotherapies presents a common problem for patients with metastatic UC. Gemcitabine and carboplatin (GCarbo) regimens are used as an option for first-line therapy in cisplatin-ineligible patients with metastatic UC. The median OS appeared comparable to that reported for the MVAC and GC combination regimens [5]. In addition, the maximum cycle number for platinum-based first-line chemotherapy is conventionally targeted at six, although this is an arbitrary number, in the first-line setting of locally advanced unresectable or metastatic UC $[6,7]$.

The advent of immune checkpoint inhibitors (ICls) has recently revolutionized the treatment of several hematological and solid malignancies. Pembrolizumab, a highly selective, humanized monoclonal IgG4k isotype antibody against PD-1, is currently the only ICl approved in Japan (since December 2017) for treating platinum-refractory UC based on the results of a phase III, open-label, randomized trial (KEYNOTE-045 trial) [8, 9].

However, the ideal number of cycles of prior chemotherapy and the timing of switching from chemotherapy to pembrolizumab remain unclear. Therefore, in practice, some patients who respond to chemotherapy continue chemotherapy until an adverse event occurs that makes it difficult to continue chemotherapy, regardless of the number of cycles.

In the present study, we retrospectively assessed the clinical outcome in patients who received platinumbased chemotherapy and pembrolizumab for metastatic UC in order to clarify the optimal timing of sequence strategies from chemotherapy to pembrolizumab.

\section{Methods}

Data from 49 patients who underwent pembrolizumab treatment after the failure of $\geq 1$ platinum-based chemotherapy regimens for advanced UC between January 2018 and August 2020 were retrospectively collected from 4 institutes. In this retrospective analysis, 34 patients who received pembrolizumab as second-line treatment after first-line platinum-based chemotherapy were examined.

All patients were pathologically diagnosed with UC [10]. Indications for the chemotherapy regimen were determined and used at each institution policy. A fixed dose of pembrolizumab ( $200 \mathrm{mg} / \mathrm{body}$ ) was intravenously infused to all patients every 3 weeks after the failure of platinum-based chemotherapy and continued until disease progression or unacceptable adverse events occurred. Computed tomography was generally performed to confirm tumor measurements before and after every four to six cycles of pembrolizumab. 
The clinicopathological data collected from medical records included the age, sex, Eastern Cooperative Oncology Group Performance Status (ECOG PS), primary tumor site, histologic testing, first-line chemotherapy regimens, number of chemotherapy cycles before pembrolizumab, hemoglobin $(\mathrm{Hb})$ concentration, neutrophil/lymphocyte ratio (NLR), levels of albumin and lactate dehydrogenase (LDH), visceral metastases (especially liver metastases) and time from previous chemotherapy. The cut-off values of the $\mathrm{Hb}$ concentration and time from previous chemotherapy were $<10 \mathrm{~g} / \mathrm{dL}$ and $<3$ months in previous studies, respectively $[8,11]$. The cut-off value of the albumin and LDH levels were set as the upper limit of the normal range $(<4.1 \mathrm{~g} / \mathrm{dl}$ and $>222$, respectively).

Because of the retrospective design of the study, the need for written informed consent was waived, and the study protocol was approved by the Ethics Committee of the Kyushu Cancer Center (Fukuoka, Japan) and complied with the 1964 Declaration of Helsinki and its later amendments.

\section{Statistical analyses}

The JMP® Pro, version 15.1.0 software package (SAS Institute, Inc., Cary, NC, USA) was used to carry out the statistical analyses. The OS was calculated from the day of the initiation of first-line treatment to the date of death from any cause and was censored at the date of the last follow-up for surviving patients, using the Kaplan-Meier method. Between-group differences in the OS were determined by the log-rank test. The Cox proportional hazards regression model was used to evaluate the significance of associations between the clinical parameters and the $\mathrm{OS}$ in univariable and multivariable analyses. $P$ values of $<0.05$ were considered to indicate statistical significance.

\section{Results}

\section{Patient characteristics}

A total of 34 patients from 4 separate institutions were included. The patient characteristics are summarized in Table 1 . The majority of the population were men $(76.5 \%, \mathrm{n}=28)$, and the median age was 71 (range, 49-87) years old. All patients received pembrolizumab after platinum-based chemotherapy for advanced UC. The first-line platinum-based chemotherapy regimen before pembrolizumab was GC in 27 patients $(79.4 \%)$ and GCarbo in 6 patients $(20.6 \%)$. Regarding the number of chemotherapy cycles before pembrolizumab, $\leq 4$ cycles were administered to 22 patients (64.7\%), $5-6$ cycles to 4 patients $(11.8 \%)$ and $\geq 7$ cycles to 8 patients (23.5\%). Sixteen patients (47.1\%) had an ECOG-PS of $\geq 1,12(35.3 \%)$ had anemia $(\mathrm{Hb}<10 \mathrm{~g} / \mathrm{dl}), 7(20.6 \%)$ had liver metastasis, $21(61.8 \%)$ had a low albumin level $(<4.1 \mathrm{~g} / \mathrm{dL}), 11$ (32.4\%) had a high LDH, and 27 had $<3$ months from previous chemotherapy (79.4\%).

\section{The OS for pembrolizumab according to the number of prior chemotherapy cycles}

The OS for pembrolizumab according to the number of prior chemotherapy cycles is shown in Fig. 1 . When the number of cycles was divided into 4 courses, the median overall survival (OS) for $\leq 4$ and $>4$ 
prior chemotherapy cycles was 7.0 months ( $95 \%$ confidence interval [Cl], 2.9 to 13.7) and 25.5 months ( $95 \% \mathrm{Cl}, 5.6$ to could not be estimated), respectively. The difference between the groups with $\leq 4$ and $>4$ prior chemotherapy cycles was statistically significant according to the log-rank test $(P=0.034)(F i g .1 \mathrm{~A})$. In addition, when the number of cycles was divided into 6 courses, the median OS for $\leq 6$ and $>6$ prior chemotherapy cycles was 11.3 months ( $95 \% \mathrm{Cl}, 5.0$ to 18.1 months) and 6.6 months ( $95 \% \mathrm{Cl}, 1.9$ to could not be estimated), respectively. The difference between the groups with $\leq 6$ and $>6$ prior chemotherapy cycles was not statistically significant according to the log-rank test $(P=0.658)$ (Fig. 1B).

Results of univariate and multivariate analyses concerning the associations between various factors and the OS with pembrolizumab treatment.

Univariate and multivariate analyses using the Cox proportional hazards model were performed to identify the prognostic factors associated with the OS in pembrolizumab treatment (Table II). The ECOGPS, LDH, liver metastases, time from previous chemotherapy and number of chemotherapy cycles before pembrolizumab $(\geq 4$, not $\geq 6$ ) were revealed to be prognostic variables in the univariate analyses.

Multivariate analyses revealed that the ECOG PS $(\geq 2$ : hazard ratio $[\mathrm{HR}] 9.994,95 \% \mathrm{Cl}=1.854-53.879, \mathrm{P}$ $=0.007$ ), liver metastases (positive: $\mathrm{HR} 3.751,95 \% \mathrm{Cl}=0.958-13.603, \mathrm{P}=0.047$ ) and time from previous chemotherapy ( $\geq 3$ months: HR $0.094,95 \% \mathrm{Cl}=0.023-0.357, \mathrm{P}<0.001)$ were independent prognostic factors, but the number of chemotherapy cycles before pembrolizumab $(\geq 4)$ was not an independent prognostic factor $(P=0.630)$.

\section{The OS from the first-line treatment according to the number of chemotherapy cycles before pembrolizumab}

The OS from the first-line treatment according to the number of chemotherapy cycles before pembrolizumab is shown in Fig. 2. When the number of cycles was divided into 4 courses, the median OS from the first-line treatment with $\leq 4$ and $>4$ prior chemotherapy cycles was 17.3 months $(95 \% \mathrm{Cl}, 12.5$ to 19.5 ) and 37.1 months ( $95 \% \mathrm{Cl}, 15.8$ to could not be estimated), respectively. The difference between the groups with $\leq 4$ and $>4$ prior chemotherapy cycles was statistically significant according to the log-rank test $(P<0.001)$ (Fig. $2 A)$. In addition, the number of cycles was divided into 6 courses, the median OS from the first-line treatment for $\leq 6$ and $>6$ prior chemotherapy cycles was 18.6 months $(95 \% \mathrm{Cl}, 13.3$ to $21.2)$ and 27.3 months $(95 \% \mathrm{Cl}, 15.9$ to 37.2$)$, respectively. The difference between the groups with $\leq 6$ and $>6$ prior chemotherapy cycles was not statistically significant according to the log-rank test $(P=$ 0.276) (Fig. 2B).

\section{Discussion}

While advanced UC clinical trials have targeted the delivery of 6 cycles of platinum-based chemotherapy, this is based on arbitrary decisions, and randomized trials have not been conducted to determine the optimal number of cycles. 
In this real-world clinical study, we retrospectively assessed the clinical outcome only in patients who received pembrolizumab as second-line treatment after platinum-based chemotherapy for metastatic UC in order to clarify the timing of sequence strategies from platinum-based chemotherapy to pembrolizumab. When the number of chemotherapy cycles was divided into 4 courses, the difference between groups receiving $\leq 4$ and $>4$ prior chemotherapy cycles was statistically significant in both the OS from pembrolizumab and the OS from the first-line chemotherapy $(p=0.034$ and $<0.001$, respectively). However, when the number of chemotherapy cycles was divided into 6 courses, the difference between groups receiving $\leq 6$ and $>6$ prior chemotherapy cycles was not statistically significant for the OS from pembrolizumab or from the first-line chemotherapy $(p=0.658$ and 0.276 , respectively).

Notably, the present study also included patients with rapidly progressive disease during $\leq 2$ cycles of first-line chemotherapy, so-called "primary resistant", who might have been refractory to immunotherapy. Judging from these results, switching treatment in cases of advanced UC from platinum-based chemotherapy to pembrolizumab should be considered after around six courses, even if no exacerbations are observed with first-line chemotherapy.

There was no internationally accepted standard of care after platinum-based chemotherapy until pembrolizumab was approved based on the KEYNOTE-045 trial results, which showed that pembrolizumab was associated with a significantly longer OS and lower rate of treatment-related adverse events than chemotherapy as second-line therapy for platinum-refractory advanced UC [8]. Therefore, urologists have been clinically administering first-line platinum-based chemotherapy regardless of the number of cycles (some patients continue the same regimen chemotherapy with a reduced volume of anticancer drugs to control adverse events (AEs) when they are responding to treatment), and various chemotherapy regimens have been used to treat patients with advanced UC as a second-line or later treatment at the discretion of each institution. However, first-line platinum-based chemotherapy rarely results in long-term disease control of advanced UC. In addition, with regard to the prognosis, the period in which the effect of pembrolizumab treatment was better than that of other chemotherapy treatments after the failure of platinum-based chemotherapy was only approximately 3 months, and the overall response rate was only $21.1 \%$; thus, the outcome remains unsatisfactory in cases of advanced UC [8,9]. Therefore, ensuring a proper timing of sequencing from chemotherapy seems important to achieve the best efficacy of both chemotherapy and pembrolizumab.

Theoretically, the timing of the sequence from chemotherapy to pembrolizumab can occur in two main patterns. One pattern involves continuing chemotherapy regardless of the number of treatment courses and only switching to pembrolizumab once progressive disease or uncontrolled adverse events occur. This pattern is almost identical to that applied in the KEYNOTE-045 clinical trial design with pembrolizumab [8]. The other pattern involves switching from chemotherapy to pembrolizumab once a certain number of courses have been reached, even if the chemotherapy did not lead to progressive disease. This pattern is almost identical to the "switch maintenance" therapy approach used in the JAVELIN Bladder 100 clinical trial design with the PD-L1 inhibitor avelumab [12]. The criteria in that study 
were eligible patients with unresectable locally advanced or metastatic UC without disease progression after four to six cycles of gemcitabine with either cisplatin or carboplatin. Maintenance avelumab plus best supportive care significantly prolonged the $\mathrm{OS}$ in all randomized patients compared to best supportive care alone (median OS of 21.4 months vs. 14.3 months; hazard ratio, $0.69 ; 95 \% \mathrm{Cl}, 0.56-0.86$; $p=0.0005)$.

One of the reasons for adopting the second pattern is to avoid unnecessary cumulative toxicity and not influence the sequence treatment by continuing chemotherapy excessively. However, AEs differ between cytotoxic chemotherapy and ICI treatment for advanced UC due to differences in the therapies' mechanisms of action. In cytotoxic chemotherapy (GC chemotherapy), severe (grade 3-4) treatmentrelated AEs included mainly hematologic toxicities (neutropenia $71.1 \%$, thrombocytopenia $57.0 \%$ and anemia 27.0\%) and nonhematologic toxicities (nausea/vomiting $22.0 \%$ and alopecia 10.5\%) [4]. In contrast, in ICl treatment (pembrolizumab after platinum-based chemotherapy), severe treatment-related AEs were less frequent than with chemotherapy in the second-line setting after platinum-based chemotherapy ( $15.0 \%$ vs. $49.4 \%)$. The most common treatment-related AEs of any grade were pruritus (19.5\% of the patients), fatigue (13.9\%) and nausea (10.9\%), and severe specific immune-related AEs were caused in $4.5 \%$ of patients. In addition, severe (grade $3-4$ ) treatment-related AEs of hematologic toxicities included anemia $0.8 \%$, and the neutrophil count decreased by $0.4 \%$. However, unexpected effects have been reported in cases of further treatment after ICls in various carcinomas, with chemotherapy as well as molecular-targeted therapy reported to demonstrate marked efficacy in this setting [13-16]. Unexpected responses to cisplatin rechallenge after treatment with ICls have been also reported in patients with metastatic UC refractory to platinum regimens [17]. Therefore, avoiding the excessive continuation of first-line platinum-based chemotherapy may be an important strategy from the perspective of performing salvage chemotherapy after platinum-based chemotherapy and pembrolizumab.

Chemotherapies have been shown to exert immune-reactive effects, such as the upregulation of MHC class molecules or tumor antigens, causing an increase in tumor antigen presentation $[18,19]$. In addition, chemotherapies have also been shown to decrease the number of immunosuppressive cells in the tumor microenvironment, such as regulatory $T$ cells and myeloid-derived suppressor cells, thereby increasing the helper T-cell accumulation at the tumor site [20,21]. These effects are theoretically beneficial for immunotherapeutic strategies after chemotherapy. While the appropriate sequence timing remains unclear, these effects appear to plateau after about six cycles of platinum-based chemotherapy according to our study based on real-world clinical data.

Several limitations associated with the present study warrant mention. First, the present study was evaluated retrospectively, not in a randomized trial but in real-world clinical practice. Second, the study population was relatively small, so it will be necessary to confirm our data in a larger study population in further studies. 
However, there are still no reports describing the optimal number of cycles of platinum-based chemotherapy before pembrolizumab for advanced UC. As a result, the findings of the present study are considered to be very important, since "the timing of switching from first-line platinum-based chemotherapy to pembrolizumab based on the data from real-world clinical practice was found to be consistent with the inclusion criteria described in the clinical trials".

\section{Conclusion}

These real-world findings indicate that when the number of chemotherapy cycles was divided into six courses, the difference between groups with $\leq 6$ and $>6$ prior chemotherapy cycles was not statistically significant for the OS with pembrolizumab therapy or the OS from the first-line chemotherapy. This finding suggests that switching treatment for advanced UC from platinum-based chemotherapy to pembrolizumab should be considered from around six cycles, even if no exacerbation is observed with first-line chemotherapy.

\section{Abbreviations}

UC: Urothelial carcinoma; OS: overall survival; HR: hazard ratio; GC: gemcitabine plus cisplatin; MVAC: cisplatin plus methotrexate, doxorubicin and vinblastine; GCarbo: Gemcitabine and carboplatin; ICl: immune checkpoint inhibitor; $\mathrm{Hb}$ : hemoglobin; NLR: neutrophil/lymphocyte ratio; LDH: lactate dehydrogenase; $\mathrm{Cl}$ : confidence interval; $\mathrm{AE}$ : adverse event

\section{Declarations}

\section{Ethics approval and consent to participate}

The present study was approved by the Institutional Review Board of National Hospital Organization Kyushu Cancer Center (2014-99), and written informed consent was obtained from the patient.

\section{Consent for publication}

Not applicable.

\section{Availability of data and materials}

The datasets used and/or analyzed during the current study are available from the corresponding author on reasonable request.

\section{Competing interests}

The authors declare that they have no conflict of interest for this study.

\section{Funding}


No funding was received.

\section{Authors' contributions}

NF, TN and KK designed the study. FM, TT, YH and AM extracted the data. NF, NN, YS and NT assisted with the data processing and statistical analysis. NF and TN wrote the article. MN and KK supervised the study and critically reviewed the manuscript. All authors have read and approved the final version of the manuscript.

\section{Acknowledgements}

Not applicable.

\section{References}

1. Necchi A, Pond GR, Raggi D, Giannatempo P, Vogelzang NJ, et al. Efficacy and safety of gemcitabine plus either taxane or carboplatin in the first- line setting of metastatic urothelial carcinoma: A systematic review and meta- analysis. Clin. Genitourin. Cancer 2016;15:331-40.

2. Sternberg CN, Mulder PD, Schornagel JH, Theodore C, Fossa SD, et al. Seven year update of an EORTC phase III trial of high-dose intensity M-VAC chemotherapy and G-CSF versus classic M-VAC in advanced urothelial tract tumours. Eur J Cancer. 2006;42:50-4.

3. Galsky MD, Chen GJ, Oh WK, Bellmunt J, Roth BJ, et al. Comparative effectiveness of cisplatin-based and carboplatin-based chemotherapy for treatment of advanced urothelial carcinoma. Ann Oncol. 2012;23:406-10.

4. Maase HDV, Hansen SW, Roberts JT, Dogliotti L, Oliver T, et al. Gemcitabine and cisplatin versus methotrexate, vinblastine, doxorubicin, and cisplatin in advanced or metastatic bladder cancer: results of a large, randomized, multinational, multicenter, phase III study. J Clin Oncol. 2000;18:306877.

5. Bamias A, Moulopoulos LA, Koutras A, Aravantinos G, Fountzilas G, et al. The combination of gemcitabine and carboplatin as first-line treatment in patients with advanced urothelial carcinoma. A Phase II study of the Hellenic Cooperative Oncology Group. Cancer. 2006;106:297-303.

6. Sonpavde GP, Mariani L, Vullo SL, Raggi D, Giannatempo P, et al. Impact of the Number of Cycles of Platinum Based First Line Chemotherapy for Advanced Urothelial Carcinoma. J Urol. 2018;200:120714.

7. NCCN Clinical Practice Guidelines in Oncology, https://www.nccn.org/professionals/physician_gls/pdf/bladder.pdf; [accessed 25 August 2020] 2020.

8. Bellmunt J, Wit RD, Vaughn D, Fradet Y, Lee JL, et I. Pembrolizumab as Second Line Therapy for Advanced Urothelial Carcinoma. N Engl J Med. 2017;376:1015-1026. 
9. Yuasa T, Urakami S, Yonese J. Recent advances in medical therapy for metastatic urothelial cancer. Int J Clin Oncol. 2018;23:599-607.

10. Moch H, Cubilla AL, Humphrey PA, Reuter VE, Ulbright TM, et al. The 2016 WHO Classification of Tumours of the Urinary System and Male Genital Organs-Part A: Renal, Penile, and Testicular Tumours. Eur Urol. 2016;70:93-105.

11. Su YL, Hsieh MC, Chiang PH, Sung MT, Lan J, et al. Novel Inflammation-Based Prognostic Score for Predicting Survival in Patients with Metastatic Urothelial Carcinoma. PLoS One. 2017;12:e0169657.

12. Powles T, Park SH, Voog E, Caserta C, Valderrama BP, et al. Avelumab Maintenance Therapy for Advanced or Metastatic Urothelial Carcinoma. N Engl J Med. 2020;383:1218-30.

13. Schvartsman G, Peng SA, Bis G, Lee JJ, Benveniste MFK, et al. Response rates to single-agent chemotherapy after exposure to immune checkpoint inhibitors in advanced non-small cell lung cancer. Lung Cancer. 2017;112:90-5.

14. Park SE, Lee SH, Ahn JS, Ahn MJ, Park K, et al. Increased Response Rates to Salvage Chemotherapy Administered after PD-1/PD-L1 Inhibitors in Patients with Non-Small Cell Lung Cancer. J Thorac Oncol. 2018;13:106-11.

15. Saleh K, Daste A, Martin N, Pons-Tostivint E, Auperin A, et al. Response to salvage chemotherapy after progression on immune checkpoint inhibitors in patients with recurrent and/or metastatic squamous cell carcinoma of the head and neck. Eur J Cancer. 2019;121:123-9.

16. Azuma T, Sugihara T, Honda S, Yoshizaki U, Niimi F, et al. Metastatic renal cell carcinoma regains sensitivity to tyrosine kinase inhibitor after nivolumab treatment: A case report. Oncol Lett. 2019;17:4011-5.

17. Gravis G, Billon E, Baldini C, Massard C, Hilgers W, et al. Unexpected response to cisplatin rechallenge after immune checkpoint inhibitors in patients with metastatic urothelial carcinoma refractory to platinum regimen. Eur J Cancer. 2018;104:236-8.

18. Ohtsukasa S, Okabe S, Yamashita H, Iwai T, Sugihara K. Increased expression of CEA and MHC class $\mathrm{I}$ in colorectal cancer cell lines exposed to chemotherapy drugs. J Cancer Res Clin Oncol. 2003;129:719-26.

19. Wan S, Pestka S, Jubin RG, Lyu YL, Tsai YC, et al. Chemotherapeutics and radiation stimulate MHC class I expression through elevated interferon-beta signaling in breast cancer cells. PLoS One. 2012;7:e32542.

20. Suzuki E, Kapoor V, Jassar AS, Kaiser LR, Albelda SM. Gemcitabine selectively eliminates splenic Gr$1+/ C D 11 b+$ myeloid suppressor cells in tumor-bearing animals and enhances antitumor immune activity. Clin Cancer Res. 2005;11:6713-21.I

21. Ghiringhelli F, Larmonier N, Schmitt E, Parcellier A, Cathelin D, et al. CD4+CD25+ regulatory T cells suppress tumor immunity but are sensitive to cyclophosphamide which allows immunotherapy of established tumors to be curative. Eur J Immunol. 2004;34:336-44.

\section{Tables}


Due to technical limitations, table 1 and 2 is only available as a download in the Supplemental Files section.

\section{Figures}

1A

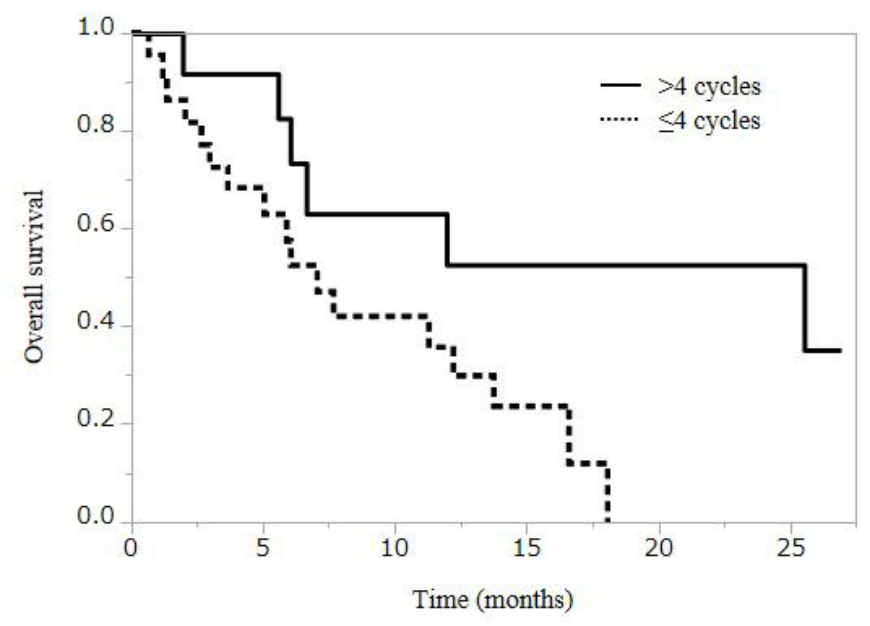

1B

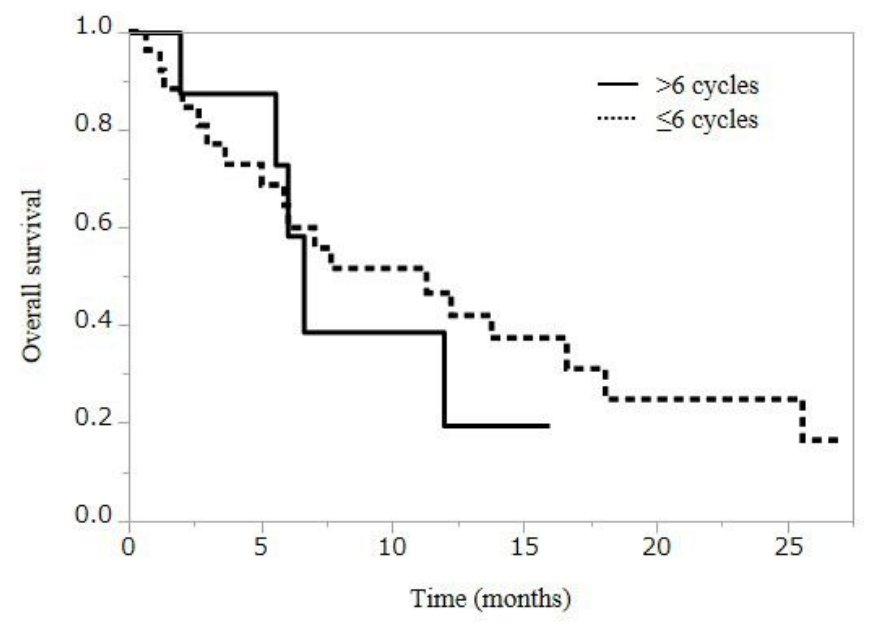

\section{Figure 1}

When the number of cycles was divided into 4 courses, the median overall survival (OS) for $\leq 4$ and $>4$ prior chemotherapy cycles was 7.0 months ( $95 \%$ confidence interval [Cl], 2.9 to 13.7 ) and 25.5 months ( $95 \% \mathrm{Cl}, 5.6$ to could not be estimated), respectively. The difference between the groups with $\leq 4$ and $>4$ prior chemotherapy cycles was statistically significant according to the log-rank test $(P=0.034)$ (Figure 1A). In addition, when the number of cycles was divided into 6 courses, the median os for $\leq 6$ and $>6$ prior chemotherapy cycles was 11.3 months ( $95 \% \mathrm{Cl}, 5.0$ to 18.1 months) and 6.6 months ( $95 \% \mathrm{Cl}, 1.9$ to could not be estimated), respectively. The difference between the groups with $\leq 6$ and $>6$ prior chemotherapy cycles was not statistically significant according to the log-rank test $(P=0.658)$ (Figure 1B).

\section{A}

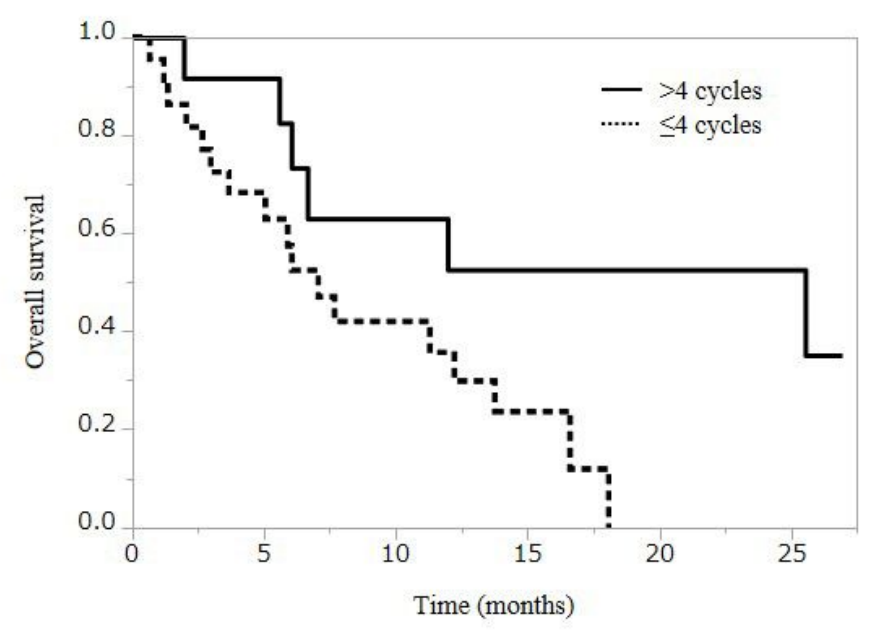

1B

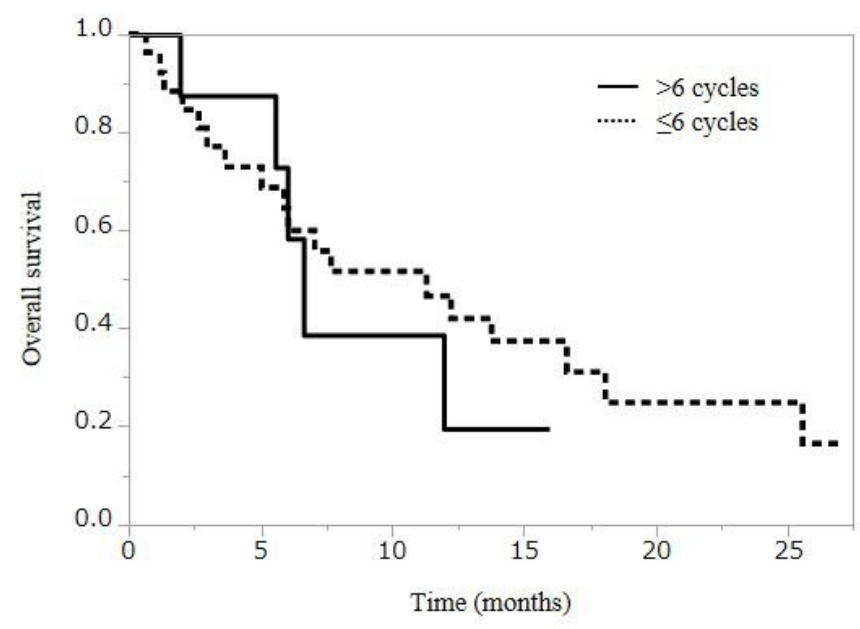


When the number of cycles was divided into 4 courses, the median overall survival (OS) for $\leq 4$ and $>4$ prior chemotherapy cycles was 7.0 months ( $95 \%$ confidence interval [Cl], 2.9 to 13.7 ) and 25.5 months ( $95 \% \mathrm{Cl}, 5.6$ to could not be estimated), respectively. The difference between the groups with $\leq 4$ and $>4$ prior chemotherapy cycles was statistically significant according to the log-rank test $(P=0.034)$ (Figure $1 \mathrm{~A})$. In addition, when the number of cycles was divided into 6 courses, the median OS for $\leq 6$ and $>6$ prior chemotherapy cycles was 11.3 months $(95 \% \mathrm{Cl}, 5.0$ to 18.1 months) and 6.6 months $(95 \% \mathrm{Cl}, 1.9$ to could not be estimated), respectively. The difference between the groups with $\leq 6$ and $>6$ prior chemotherapy cycles was not statistically significant according to the log-rank test ( $P=0.658)$ (Figure 1B).

2A

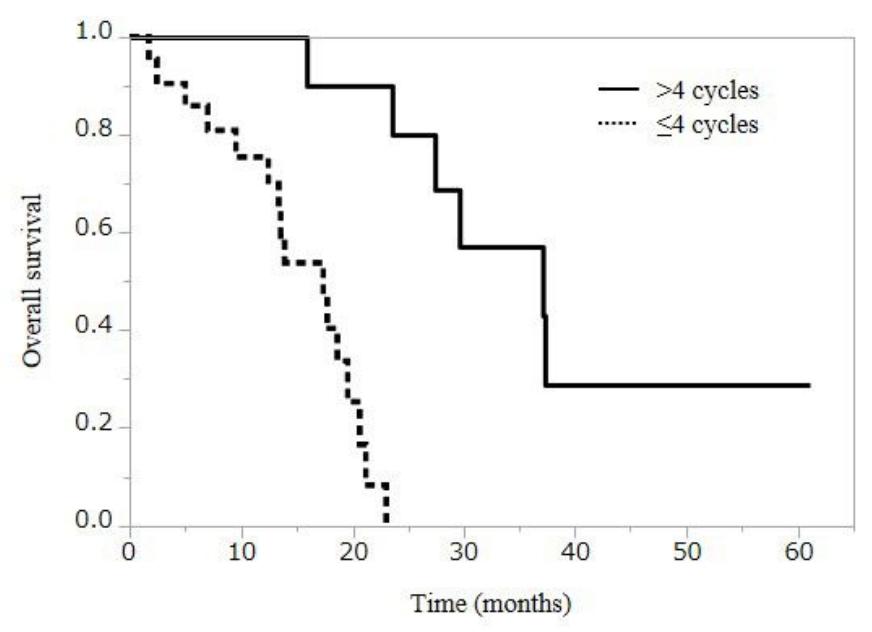

2B

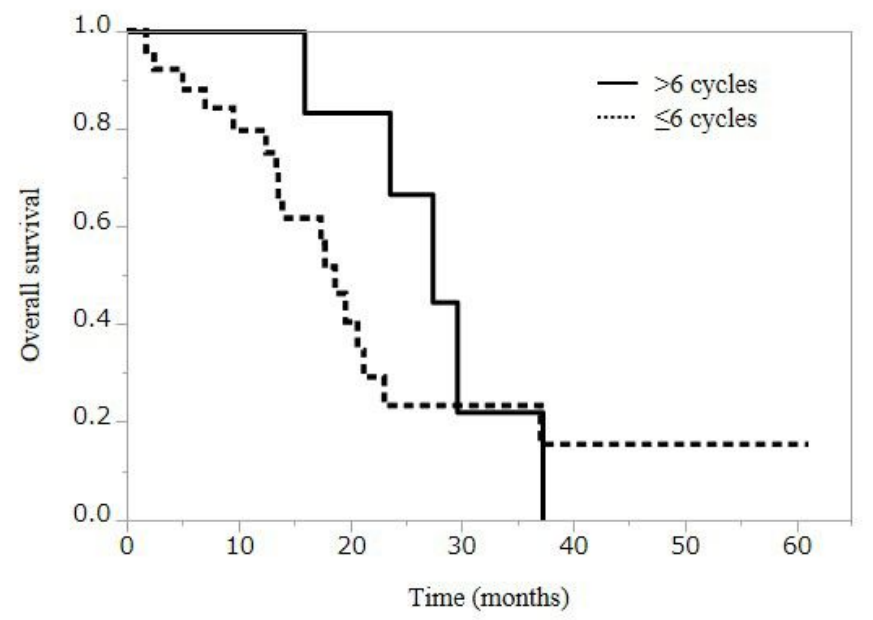

\section{Figure 2}

The OS from the first-line treatment according to the number of chemotherapy cycles before pembrolizumab is shown in Figure 2. When the number of cycles was divided into 4 courses, the median OS from the first-line treatment with $\leq 4$ and $>4$ prior chemotherapy cycles was 17.3 months $(95 \% \mathrm{Cl}, 12.5$ to 19.5$)$ and 37.1 months ( $95 \% \mathrm{Cl}, 15.8$ to could not be estimated), respectively. The difference between the groups with $\leq 4$ and $>4$ prior chemotherapy cycles was statistically significant according to the logrank test $(P<0.001)$ (Figure $2 A)$. In addition, the number of cycles was divided into 6 courses, the median OS from the first-line treatment for $\leq 6$ and $>6$ prior chemotherapy cycles was 18.6 months $(95 \% \mathrm{Cl}, 13.3$ to 21.2$)$ and 27.3 months $(95 \% \mathrm{Cl}, 15.9$ to 37.2$)$, respectively. The difference between the groups with $\leq 6$ and $>6$ prior chemotherapy cycles was not statistically significant according to the log-rank test $(P=0.276)$ (Figure 2B). 
2A

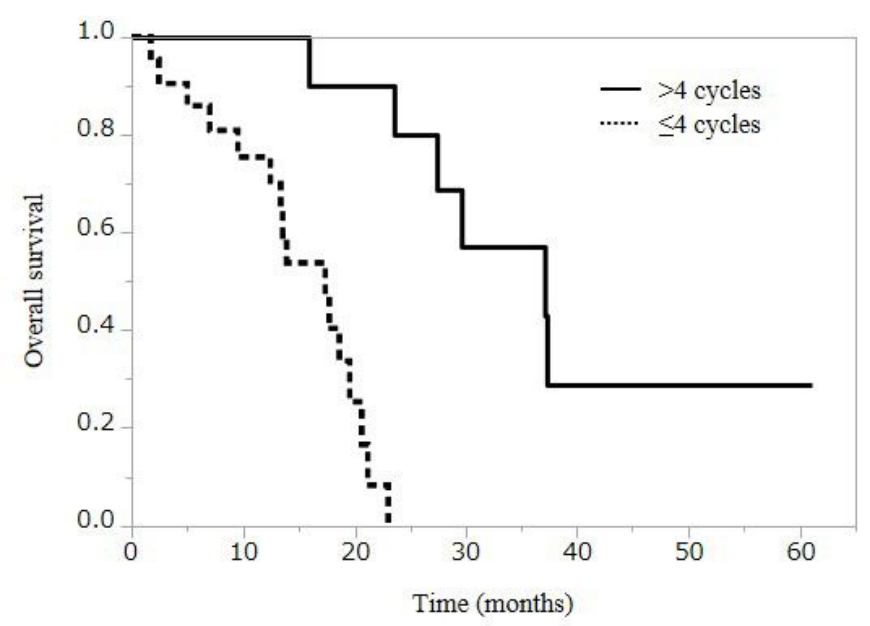

2B

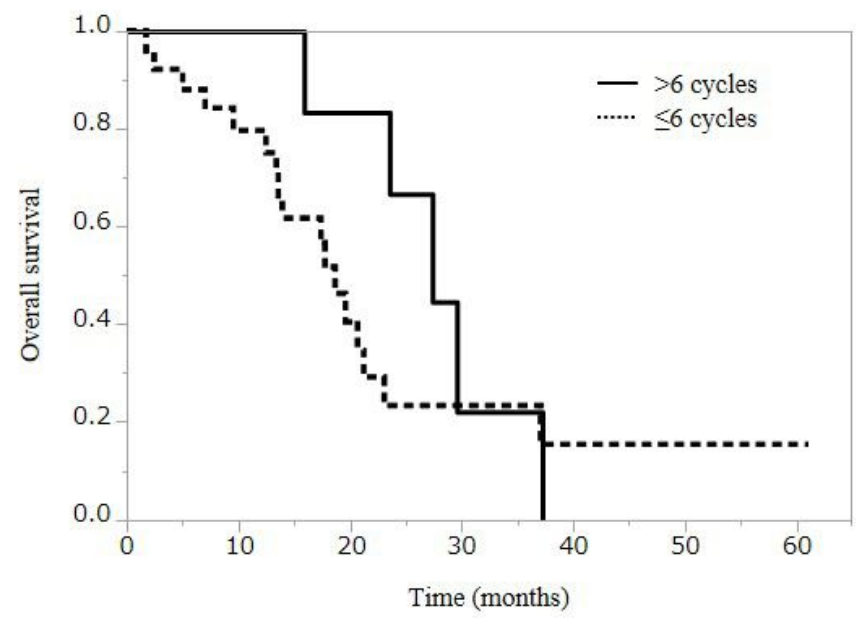

\section{Figure 2}

The OS from the first-line treatment according to the number of chemotherapy cycles before pembrolizumab is shown in Figure 2. When the number of cycles was divided into 4 courses, the median OS from the first-line treatment with $\leq 4$ and $>4$ prior chemotherapy cycles was 17.3 months $(95 \% \mathrm{Cl}, 12.5$ to 19.5 ) and 37.1 months ( $95 \% \mathrm{Cl}, 15.8$ to could not be estimated), respectively. The difference between the groups with $\leq 4$ and $>4$ prior chemotherapy cycles was statistically significant according to the logrank test $(P<0.001)$ (Figure $2 A)$. In addition, the number of cycles was divided into 6 courses, the median OS from the first-line treatment for $\leq 6$ and $>6$ prior chemotherapy cycles was 18.6 months $(95 \% \mathrm{Cl}, 13.3$ to 21.2 ) and 27.3 months $(95 \% \mathrm{Cl}, 15.9$ to 37.2$)$, respectively. The difference between the groups with $\leq 6$ and $>6$ prior chemotherapy cycles was not statistically significant according to the log-rank test $(P=0.276)$ (Figure 2B).

\section{Supplementary Files}

This is a list of supplementary files associated with this preprint. Click to download.

- Table1.xls

- Table1.xls

- Table2.xls

- Table2.xls 